\title{
Cas de chikungunya liés aux voyages au Canada, 2014
}

\author{
Drebot MA ${ }^{1 *}$, Holloway $\mathrm{K}^{1}$, Zheng $\mathrm{H}^{2}$, Ogden $\mathrm{NH}^{2}$ \\ ${ }^{1}$ Laboratoire national de microbiologie, Agence de la santé publique du Canada, Winnipeg (Manitoba) \\ ${ }^{2}$ Centre des maladies infectieuses d'origine alimentaire, environnementale et zoonotique, Agence de la santé publique du \\ Canada, Ottawa (Ontario)
}

*Auteur-ressource : $\underline{\text { mike.drebot@phac-aspc.gc.ca }}$

\section{Résumé}

Depuis le printemps 2014, le nombre de cas diagnostiqués de chikungunya liés aux voyages a considérablement augmenté au Canada. Le 9 décembre 2014, on comptait 320 cas confirmés et 159 cas probables au pays, la majorité des provinces ayant détecté au moins un cas importé. Cette augmentation subite des cas d'infection a été associée à l'arrivée du virus du chikungunya aux Caraïbes et à sa propagation dans les Amériques. Les éclosions qui sévissent actuellement dans la région Asie-Pacifique ont également contribué à l'importation de cas par des voyageurs canadiens. Une sensibilisation renforcée des cliniciens à l'infection à chikungunya est essentielle à la pose du diagnostic. Cette situation souligne la nécessité d'interroger toute personne présentant une fièvre ou l'apparition récente d'une polyarthralgie sur ses antécédents de voyage et d'envisager la soumission d'échantillons aux laboratoires provinciaux et au Laboratoire national de microbiologie afin de rechercher la présence du virus du chikungunya et d'autres maladies, le cas échéant. Il est également essentiel d'informer sans relâche les voyageurs concernant l'utilisation de mesures préventives destinées à diminuer le risque d'exposition aux moustiques lors de voyages dans des régions d'endémicité.

\section{Introduction}

Le chikungunya est une maladie virale transmise par les moustiques qui jusque récemment n'était endémique que dans certains pays d'Afrique, d'Asie et des océans Indien et Pacifique. En décembre 2013, la détection confirmée de deux cas de chikungunya non importés aux Caraïbes, dans l'île de Saint-Martin/Sint Maarten, a été signalée à l'Organisation panaméricaine de la Santé (OPS) (1). Ces cas ont marqué l'arrivée du virus du chikungunya dans l'hémisphère occidental. Durant 2014, des cas de transmission locale du virus ont été détectés dans plus de 40 pays ou territoires de la Caraïbe, de l'Amérique centrale, de l'Amérique du Sud, du Mexique et des États-Unis $(2,3)$ (Tableau 1).

Tableau 1 : Pays et territoires où des cas de chikungunya transmis localement/autochtones ont été signalés $(2,4)$.

\begin{tabular}{|l|l|l|l|}
\hline \multicolumn{1}{|c|}{ Afrique } & \multicolumn{2}{c|}{ Amériques } & \multicolumn{1}{c|}{ Asie } \\
\hline Afrique du Sud & Guyane & Montserrat & Bangladesh \\
\hline Bénin & Anguilla & Nicaragua & Bhoutan \\
\hline Burundi & Antigua-et-Barbuda & Panama & Cambodge \\
\hline Cameroun & Aruba & Saint Barthélemy & Inde \\
\hline Comores & Barbade & Saint-Martin & Malaisie \\
\hline Gabon & $\begin{array}{l}\text { Îles Vierges } \\
\text { britanniques }\end{array}$ & Saint-Vincent-et-les-Grenadines & Maldives \\
\hline Guinée & Îles Caïmans & Sainte-Lucie & \\
\hline Guinée équatoriale & Brésil & Sint Maarten & Laos \\
\hline Kenya & Colombie & Suriname & Myanmar (Birmanie) \\
\hline Madagascar & Costa Rica & Trinité-et-Tobago & Pakistan \\
\hline Malawi & Curaçao & Îles Turks et Caicos & Philippines \\
\hline Maurice & Dominique & Îles Vierges des États-Unis & Singapour \\
\hline Mayotte & $\begin{array}{l}\text { République } \\
\text { dominicaine }\end{array}$ & & \\
\hline
\end{tabular}




\begin{tabular}{|l|l|l|l|}
\hline Ouganda & Martinique & Porto Rico & Chine \\
\hline République centrafricaine & Bahamas & Saint-Kitts-et-Nevis & Indonésie \\
\hline République démocratique du Congo & Belize & États-Unis (Floride) & Thaïlande \\
\hline République du Congo & El Salvador & Venezuela & Timor \\
\hline Réunion & Guyane française & & Vietnam \\
\hline Sénégal & Grenade & & Yémen \\
\hline Seychelles & Guatemala & & \\
\hline Sierra Leone & Guadeloupe & Océanie/îles du Pacifique & Europe \\
\hline Soudan & Haïti & États fédérés de Micronésie & France \\
\hline Tanzanie & Jamaïque & Nouvelle-Calédonie & Italie \\
\hline Zimbabwe & Mexique & Papouasie-Nouvelle-Guinée & \\
\hline
\end{tabular}

Jusqu'à présent, aucune transmission locale du virus du chikungunya n'a été observée au Canada, vraisemblablement en raison de l'absence des principaux moustiques vecteurs que sont Aedes aegypti et Aedes albopictus. Les Canadiens effectuent cependant chaque année plus de 2,5 millions de voyages à destination des Caraïbes (5) et sont également très nombreux à se rendre dans la région Asie-Pacifique, région où le nombre d'éclosions persistantes du virus du chikungunya et d'autres agents transmis par les moustiques est en hausse et où certains cas canadiens ont été contractés $(6,7)$. L'objet de cet article est de passer en revue la maladie, d'examiner l'accroissement considérable du nombre de pays qui déclarent désormais des cas de virus du chikungunya et de signaler l'augmentation des cas de virus liés aux voyages diagnostiqués au Canada en 2014 par rapport aux années précédentes.

\section{Tableau clinique}

Les symptômes se manifestent généralement trois à sept jours après la piqûre d'un moustique infecté, habituellement sous la forme d'une fièvre et d'une polyarthralgie d'apparition brutale $(8,9)$. Les douleurs articulaires sont généralement symétriques, touchant habituellement les mains et les pieds, et peuvent être invalidantes. L'apparition d'une éruption cutanée, de céphalées, d'une conjonctivite, de nausées et de fatigue est également possible. L'analyse des paramètres biologiques révèle souvent une lymphocytopénie, une thrombocytopénie et une élévation du taux de créatinine et de transaminases hépatiques (2). Le diagnostic différentiel avec la dengue (9) est le plus courant; des cas d'infections concomitantes par les virus de la dengue et du chikungunya ont toutefois également été signalés (10). Les symptômes disparaissent généralement spontanément au bout de deux à trois jours; la douleur articulaire peut toutefois persister pendant plusieurs semaines, voire plusieurs mois. Si le rétablissement est complet pour la plupart des patients, quelques cas de complications oculaires, neurologiques et cardiaques ont été signalés. Le traitement est un traitement de soutien visant à soulager les symptômes; il n'existe actuellement aucun vaccin.

\section{Diagnostic en laboratoire}

En présence de cas suspects concernant des voyageurs canadiens, il convient de réaliser des tests de détection des anticorps anti-chikungunya IgM ainsi que des épreuves biologiques visant à confirmer la présence d'anticorps neutralisants spécifiques $(2,11)$. Les voyageurs qui tombent malades à leur retour immédiat d'un voyage peuvent également être soumis à un test de détection de l'acide ribonucléique (ARN) viral selon une technique de réaction en chaîne de la polymérase (PCR), la virémie pouvant persister pendant une semaine ou plus. L'isolement du virus est également à envisager pour les cas aigus. Pour l'instant, tous les tests concernant le virus du chikungunya sont effectués au Laboratoire national de microbiologie (LNM). II existe aussi désormais des trousses commerciales d'essai immuno-enzymatique (ELISA) pour le dosage des anticorps IgM; ces trousses sont en cours de validation par les Centers for Disease Control and Prevention (CDC) des États-Unis et le LNM à des fins d'utilisation en tant que tests de « première ligne ».

En raison du chevauchement de symptômes, le diagnostic différentiel doit s'intéresser non seulement au chikungunya, mais aussi à d'autres maladies transmises par les moustiques, notamment la dengue; il convient également d'envisager des tests de dépistage du paludisme, du virus Zika et du virus de l'encéphalite japonaise, en fonction des antécédents de voyage des patients. 


\section{Épidémiologie du chikungunya au Canada et dans les Amériques}

Au 29 décembre 2014, le nombre de cas suspects de transmission locale signalés par l'OPS s'élevait à plus d'un million, dont près de 23000 ont été confirmés en laboratoire $(2,4)$. Les États-Unis ont signalé 2320 cas importés et la détection en Floride de 11 cas de transmission locale.

Le chikungunya n'est pas une maladie à déclaration obligatoire au Canada, mais le nombre de cas identifiés par les résultats des analyses diagnostiques soumises au LNM donne une indication du nombre de Canadiens touchés par le virus. Les années précédentes, on comptait entre 1 et 20 cas par an sur environ 200 demandes d'analyses diagnostiques.

Au 9 décembre 2014, 320 cas confirmés et 159 cas probables (IgM positif, test de confirmation à venir) avaient été identifiés au Canada en laboratoire parmi des voyageurs de retour de régions touchées des Amériques et de la région Asie-Pacifique (Figure 1). De plus, pour le mois de décembre 2014, il y avait plus de 100 cas suspects qui sont en voie de subir un test de dépistage sérologique.

En 2014, le nombre d'échantillons sériques soumis à des fins d'analyse a augmenté à plus de 1800 , ce qui témoigne à la fois d'une prise de conscience renforcée de l'éclosion du virus et d'une augmentation du nombre de cas suspects présentant des symptômes cliniques évoquant le chikungunya.

Figure 1 : Nombre de cas de chikungunya liés aux voyages diagnostiqués au Canada de janvier 2008 au 9 décembre 2014

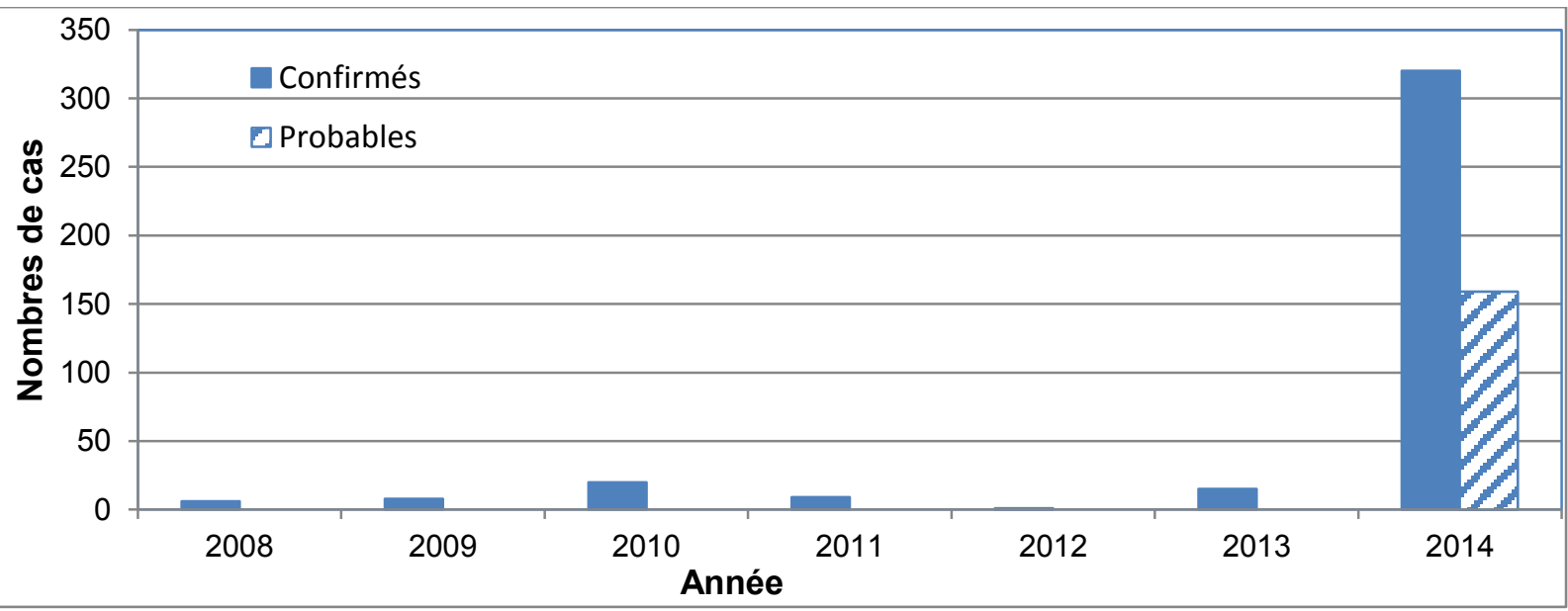

La majorité des cas dont les antécédents de voyage ont été documentés s'étaient rendus aux Caraïbes, région qui connaît une transmission virale intense depuis le printemps 2014. Le premier cas confirmé lié à un voyage aux Caraïbes a été détecté chez un résident du Québec qui s'était rendu en Martinique début janvier 2014 et était rentré au Canada début février (C. Therrien et M. Drebot, communication personnelle, 2014).

La majorité des provinces comptaient quelques cas (Québec 114, Ontario165, Alberta 14 et Colombie-Britannique 14, Manitoba 7, Saskatchewan, Nouveau-Brunswick et Terre-Neuve-et-Labrador, < de 5 cas chacune). En outre, chez environ $20 \%$ des patients testés, l'existence d'une virémie avait été révélée par la détection d'ARN viral dans les échantillons sériques selon une technique de réaction en chaîne de la polymérase. Si des moustiques vecteurs appartenant à l'espèce Aedes capables de transmettre la maladie venaient à s'établir dans l'une des provinces, cela aurait des implications pour la transmission locale au pays.

\section{Conclusion}

Au cours de l'année 2014, le nombre de cas de chikungunya détectés au Canada a connu une rapide augmentation. Les 320 cas confirmés et les 159 cas probables (au 9 décembre 2014) représentent de loin le plus grand nombre de cas de chikungunya jamais documentés en un an au pays. Il est probable que ce chiffre soit 
une sous-estimation de la réalité, en raison des erreurs de diagnostic et de la non-détection de certains cas légers de la maladie.

Un examen plus approfondi des antécédents de voyage des patients nous permettra de mieux cerner les sources d'infection par le chikungunya des voyageurs canadiens. II est probable que l'augmentation de cas observée en 2014 était associée aux voyages à destination des Caraïbes, bien que l'éclosion persistante qui touche la région Asie-Pacifique puisse y avoir contribué.

Le chikungunya n'est pas une maladie à déclaration obligatoire au Canada, mais les cas détectés par les épreuves de confirmation en laboratoire permettent d'en suivre les effets. Les patients présentant des symptômes cliniques évoquant le chikungunya à leur retour d'un voyage dans des pays où circule le virus doivent subir des épreuves visant à déterminer s'ils ont été exposés au virus. L'algorithme de diagnostic en laboratoire fait appel à des techniques de diagnostic sérologique et moléculaire pour identifier les patients ayant contracté la maladie. Les échantillons sériques de cas aigus sont analysés à la recherche d'anticorps IgM; les échantillons positifs sont ensuite analysés à la recherche d'anticorps neutralisants spécifiques du virus concerné et d'ARN viral. La soumission des sérums en phase de convalescence est encouragée. En effet, les échantillons en phase de convalescence testés permettront de documenter les séroconversions; de plus, les sérums en phase aiguë provenant de cas suspects pourraient ne pas contenir des niveaux mesurables de $\operatorname{lgM}$ et $\lg C$ qui seraient détectables dans un échantillon de sérum subséquent.

À la lumière de l'augmentation des cas de chikungunya observée en 2014 au Canada, une sensibilisation renforcée des voyageurs et des cliniciens aux risques posés par les maladies à transmission vectorielle et à la prévention de l'infection est bien fondée. Les mesures préventives sont les mêmes que celles utilisées pour la prévention de toutes les maladies transmises par les moustiques (12).

Le Gouvernement du Canada a émis des conseils de santé aux voyageurs sur le chikungunya, leur recommandant de se protéger des piqûres de moustiques lorsqu'ils se rendent dans des régions potentiellement touchées par le virus. En cas d'apparition de symptômes, en cours de voyage ou une fois de retour au Canada, il est conseillé aux voyageurs de communiquer avec un professionnel de la santé et de lui indiquer les régions où ils ont voyagé ou séjourné (13).

\section{Remerciements}

Les auteurs tiennent à remercier les laboratoires de santé provinciaux canadiens d'avoir bien voulu mettre à leur disposition les chiffres de déclaration de cas ainsi que Kristina Dimitrova, Kai Makowski, Phillip Snarr et Maya Andonova du soutien technique en laboratoire qu'ils ont bien voulu leur accorder durant leur investigation. De plus, nous remercions Dr Robbin Lindsay pour sa lecture critique du manuscript et ses précieux commentaires par rapport à son contenu.

\section{Conflit d'intérêts}

Aucun

\section{Références}

(1) Omarjee R, Prat C, Flusin O, Boucau S, Tenebray B, Merle O, Huc-Anais P, Cassadou S, Leparc-Goffart I. Importance of case definition to monitor ongoing outbreak of chikungunya virus on a background of actively circulating dengue virus, St Martin, December 2013 to January 2014. Eurosurveillance. 2014; 19: pii: 20753.

(2) Pan American Health Organization. Number of reported cases of chikungunya fever in the Americas-EW 40 (October 24, 2014). http://www.paho.org/hq/index.php?option=com_topics\&view=article\&id=343\&ltemid=40931

(3) Powers AM. Risks to the Americas associated with the continued expansion of Chikungunya virus. J Gen Virol. 2014 Sep 19. pii: vir.0.070136-0. doi: 10.1099/vir.0.070136-0.

(4) Centers for Disease Control and Prevention. Countries and territories where chikungunya cases have been reported (as of June 17, 2014). http://www.cdc.gov/chikungunya/pdfs/ChikungunyaMap.pdf 
(5) Statistique Canada. Enquête sur les voyages internationaux (résidents canadiens), 2012.

(6) Roth A, Mercier A, Lepers C, Hoy D, Duituturaga S, Benyon E, Guillaumot L, Sourares Y. Concurrent outbreaks of dengue, chikungunya, and Zika virus infections-An unprecedented epidemic wave of mosquito-borne viruses in the Pacific 2012-2014. Eurosurveillance 2014; 19: pil=20929.

(7) Schwartz KL, Giga A, Boggild AK. Chikungunya fever in Canada: Fever and polyarthritis in a returned traveller. CMAJ. 2014 Jul 8; 186(10):772-4. doi: 10.1503/cmaj.

(8) Morrison TE. Reemergence of chikungunya virus. J Virol. 2014; 88:11644-7.

(9) Deilgat, M., Geduld, J., Drebot, M. Éclosion de chikungunya dans les Caraïbes (2013-2014). Relevé des maladies transmissibles au Canada, janvier 2014; 40(2):7-12. http://www.phac-aspc.gc.ca/publicat/ccdr-rmtc/14vol40/dr-rm4002/dr-rm40-02-chik-fra.php

(10) Chang SF, Su CL, Shu PY, Yang CF, Liao TL, Cheng CH, Hu HC, Huang JH. Concurrent isolation of chikungunya virus and dengue virus from a patient with coinfection resulting from a trip to Singapore. J Clin Microbiol. 2010 Dec; 48(12):4586-9. doi: 10.1128/JCM.01228-10.

(11) Centers for Disease Control and Prevention. Chikungunya-Information for healthcare providers. http://www.cdc.gov/chikungunya/pdfs/CHIKV_Clinicians.pdf

(12) Comité consultatif de la médecine tropicale et de la médecine des voyages. Déclaration relative aux mesures de protection individuelle pour prévenir les piqûres ou morsures d'arthropodes. Relevé des maladies transmissibles au Canada 2012; 38(DCC-3). http://www.phac-aspc.gc.ca/publicat/ccdr-rmtc/12vol38/acs-dcc-3/index-fra.php.

(13) Conseil de santé aux voyageurs du Gouvernement du Canada. Le chikungunya : situation mondiale. http://voyage.gc.ca/voyager/sante-securite/conseils-sante-voyageurs/chikungunya-mondiale 